\title{
Effects of ABS-g-MAH on Morphologies and Properties of PC/ASA/PMMA blends
}

\author{
Ruogu Tang \\ School of Polymer Science and Engineering, Qingdao University of Science and \\ Technology. Qingdao, China. 266042
}

\begin{abstract}
In this study, ABS-g-MAH was used as compatibilizer in poly carbonate/acrylonitrile styrene acrylate/poly(methyl methacrylate) blends. The polymer blends were prepared via a two-step method, raw PC, ASA, PMMA resins and ABS-g-MAH additives were mixed and granulated by extrusion molding and then the standard samples were obtained by injection molding. A comprehensive characterizations were performed on the PC/ASA/PMMA blends of their morphologies, mechanical properties and thermal properties. The results showed that the addition of ABS-g-MAH could promote the compatibilities among PC, ASA and PMMA, and ABS-g-MAH would significantly alter the mechanical and thermal properties of blends. It could be concluded that the appropriate usage of ABS-g-MAH could bring expected benefits on PC/ASA/PMMA blends.
\end{abstract}

Key words: polymer blends, ABS-g-MAH, mechanical strength, stress, segment relaxation

Contact: ruogutang@gmail.com 


\section{Introduction}

Poly(carbonate/acrylonitrile styrene acrylate) is a relative new types of polymer blends and it has proved to be a good supplement and alternative to $\mathrm{PC} / \mathrm{ABS}^{[1-3]}$, this blend combines the advantages of polycarbonate and ASA, however, like many other polymer blends, the compatibility and related mechanical properties are not negligible ${ }^{[4,5]}$. One widely spreaded method is to use compatbilizer during the processing ${ }^{[6]}$, especially for the polar/non-polar blending polymer systems, if a polar compatibilizer introduced onto the non-polar polymer, the compatibility could be imporved ${ }^{[7]}$. But on the other hand, compatibilizers also alter the morphologies and other properties of polymer blends and they may cause different unexpected effects $^{[8-10]}$. Therefore the effects of compatibilizers should be comprehensively analyzed.

In this study, the PC/ASA/PMMA blends were prepared by extrusion and injection molding and the ABS-g-MAH was used as compatibilizer during the processing. The products were characterized of their surface morphologies, mechanical strengths and thermal stabilities, and the effects of ABS-g-MAH were investigated.

\section{Experiment}

2.1 Granulation of PC/ASA/PMMA blends

The polycarbonate (PC-110, purchased from Qimei Corp, Taiwan), acrylonitrile/styrene/acrylate (purchased from LG Corp, South Korea), poly(methyl methacrylate) (IF850, purchased from LG Corp) and ABS-g-MAH (purchased from Shenyang four-dimensional polymer plastic Ltd, China) were granulated by the twin screw extruder (SHJ-20, provided by Nanjing Giant Machinery Co.,Ltd., China) based on the ormula in Table 1. The temperatures were set as : zone $1: 230^{\circ} \mathrm{C}$, zone $2: 235^{\circ} \mathrm{C}$, zone $3: 238^{\circ} \mathrm{C}$, zone $4: 240^{\circ} \mathrm{C}$, extruder head: $240^{\circ} \mathrm{C}$, polymer melt: $220^{\circ} \mathrm{C}$.

Table 1. Formulation of PC/ASA/PMMA blend (unit: g)

\begin{tabular}{|l|l|l|l|l|}
\hline No. & PC & ASA & PMMA & ABS-g-MAH \\
\hline 1 & 70 & 20 & 10 & 0 \\
\hline 2 & 70 & 20 & 10 & 3 \\
\hline 3 & 70 & 20 & 10 & 6 \\
\hline 4 & 70 & 20 & 10 & 9 \\
\hline 5 & 70 & 20 & 10 & 12 \\
\hline 6 (Pure PC) & 100 & 0 & 0 & 0 \\
\hline
\end{tabular}

\subsection{Preparation of PC/ASA/PMMA standard test samples}

The PC/ASA/PMMA standard test samples were obtained via injection molding (TTI-130F2, Welltec Machinery Ltd, China). The temperatures were set as : injection head: $260-265^{\circ} \mathrm{C}$, zone 1 : $240-250^{\circ} \mathrm{C}$, zone $2: 245-252^{\circ} \mathrm{C}$, zone $3: 248-255^{\circ} \mathrm{C}$, zone4: $250-258^{\circ} \mathrm{C}$, mold: $85^{\circ} \mathrm{C}$. The injection pressure was set as $10 \mathrm{MPa}$, the holding pressure was set as $10.5 \mathrm{MPa}$ and the pressure was kept for 10 seconds.

\subsection{Polymer blends characterization}

Morphology investigation: Scanning electron microscope (SEM, JEOL JSM-6700F, Japan) was used to study the impact fracture surfaces of polymer blends samples, tetrahydrofuran would be used for etching to remove the ASA and PMMA components.

Mechanical properties tests: the PC/ASA/PMMA blends were characterized of the tensile 
strength (GB/T1040.1-2006), Shore $D$ hardness (GB/T2411-2008), flexural strength (GB/T9341-2008), notched impact strength (GB/T1043.1-2008, 7.5J of impact energy).

Dynamics properties test: dynamic mechanical analysis (DMA, DMTS EPLexor 500N, Germany) was used for measuring thedynamic loss modulus, test frequency was set as $5 \mathrm{~Hz}$.

Thermal properties characterization: differential scanning calorimetry (DSC, NETZSCH DSC204, Germany) was used to investigate the thermal properties, the heating/cooling rate was set as $20^{\circ} \mathrm{C} / \mathrm{min}$.

Thermal stability tests: the thermal stabilities of polymer blends were studied by Vicat softening temperature (ZWK1302-B, MTS Systems Corporation) measurement (GB/T1633-2000, heating rate: $120^{\circ} \mathrm{C} / \mathrm{h}$ ) and thermogravimetric analysis (TGA, NETZSCH TG209F1, Germany).

\section{Results and discussion}

\subsection{Morphology}

As an effective compatibilizer, ABS-g-MAH promoted the compatbilities between each components in the PC/ASA/PMMA blends. As shown in the SEM images in Figure 1, ABS-g-MAH added PC/ASA/PMMA blends had less separation phases (areas) and exhibited a more obvious IPN structures. In addition, SEM images of etched samples (Figure 2) showed that without addition of ABS-g-MAH, the PC didn't distribute evenly in the blends and it had a large aggregated area, the black hole in the SEM image indicated that there were many aggregations of ASA and PMMA components, which were removed by THF. By contrast, after adding ABS-g-MAH, the distribution of component each components improved as the black hole disappeared. The compatibilizing effect of ABS-g-MAH could be attributed to the its capacity reducing on interfacial tensions, and the ABS-g-MAH could prevent the separated components from re-aggregating, leading to a better distribution and higher homogeneity ${ }^{[11]}$.

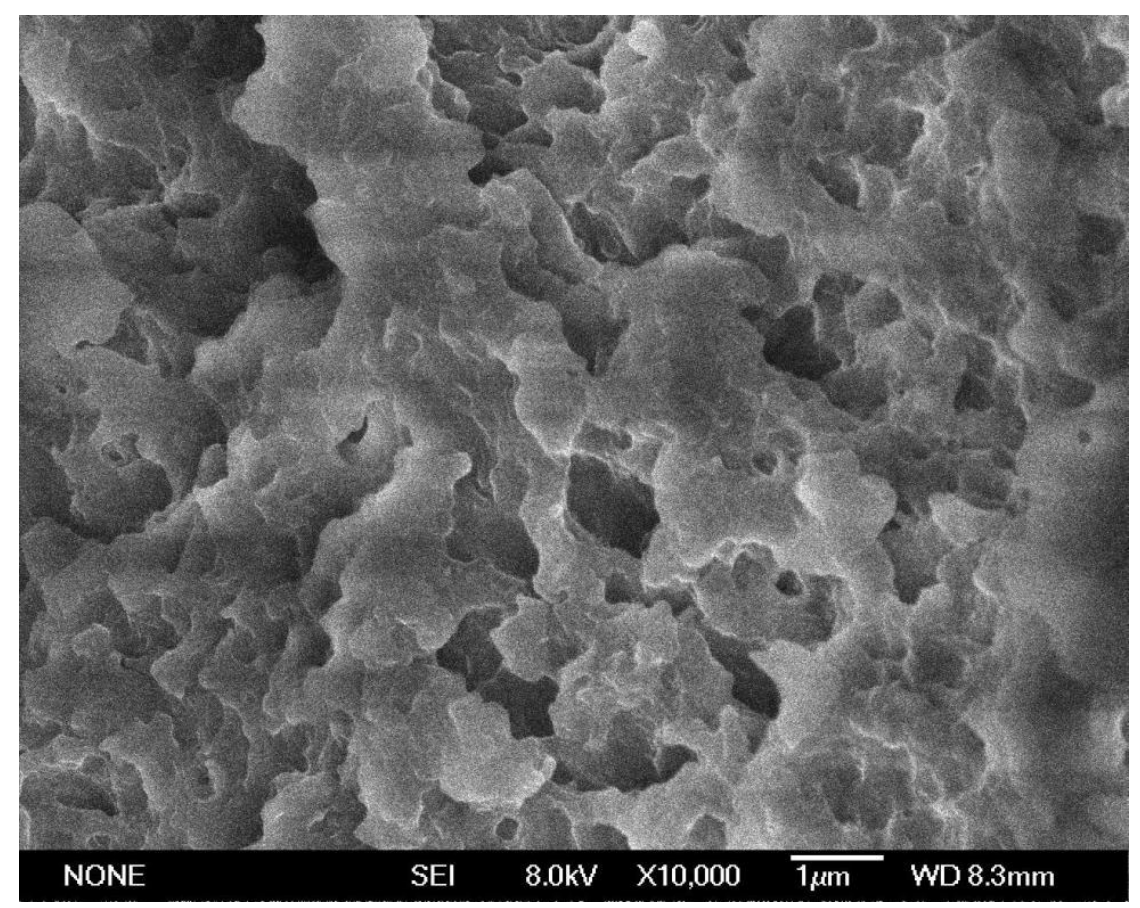

Fig.1(a). SEM images of fractures of PC/ASA/PMMA (70:20:10) 


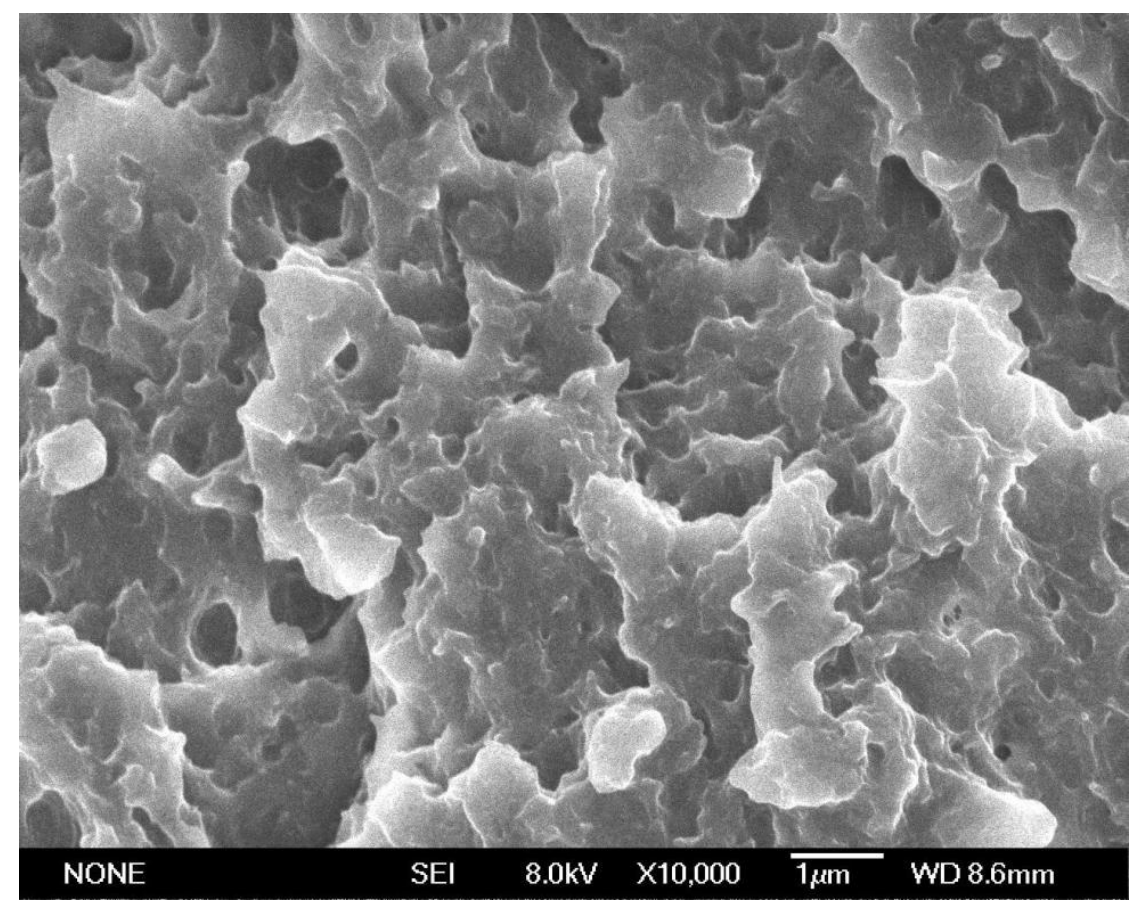

Fig.1(b). SEM images of fractures of PC/ASA/PMMA/ABS-g-MAH (70:20:10:9)

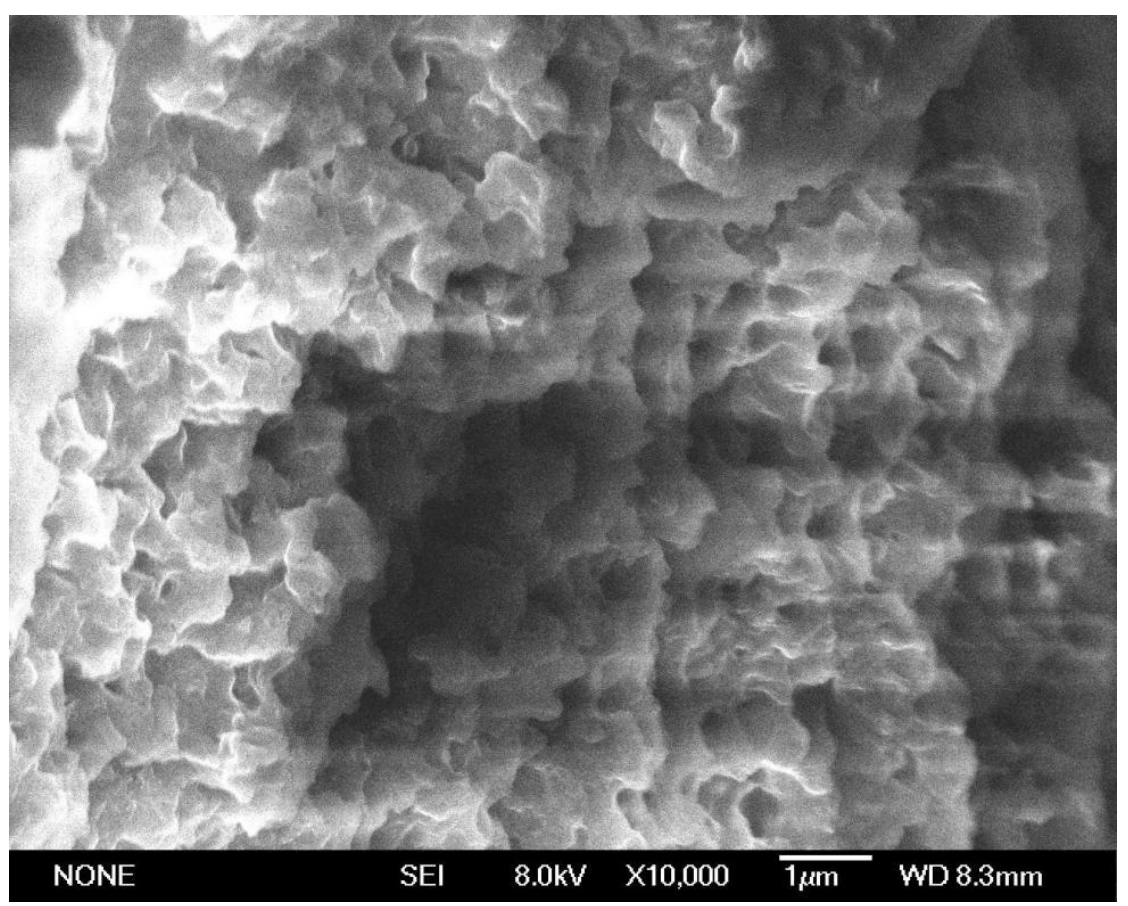

Fig.2(a). SEM images of etched fractures of PC/ASA/PMMA (70:20:10) 


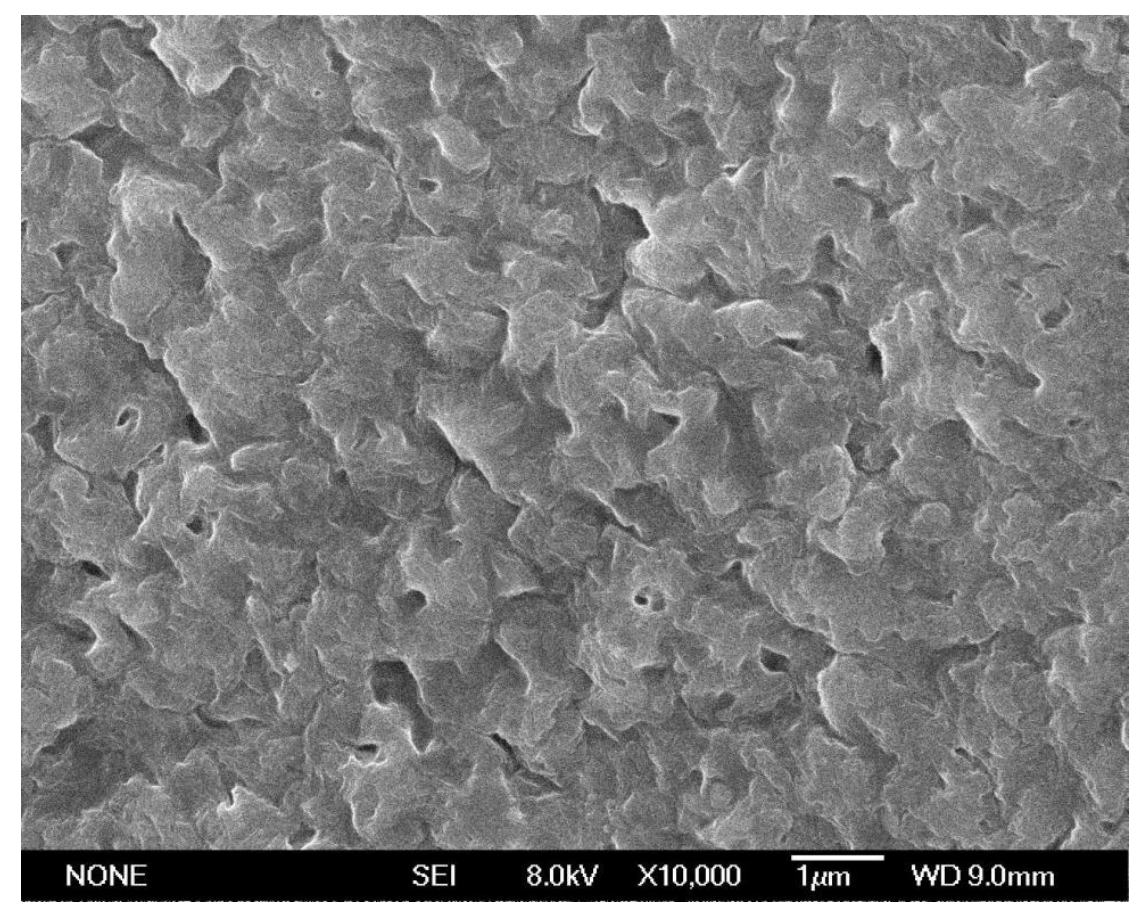

Fig.2(b). SEM images of etched fractures of PC/ASA/PMMA/ABS-g-MAH (70:20:10:9)

\subsection{Mechanical properties}

The ABS-g-MAH significantly affected on mechanical properties of PC blends, it was found that, on Table 2, the lower amount of ABS-g-MAH addition reduced the mechanical strengths of the blends, while enough ABS-g-MAH content (at 9g addition in 100) did promote the mechanical performances. This is because for muti-components based polymer blends, compatibilizer would affect in different approaches, at lower content, its compatibilizing effects could not make up its damage on blends homogeneities, which was overcome by putting enough ABS-g-MAH into the blends ${ }^{[12,13]}$.

Table 2. Effects of ABS-g-MAH on mechanical properties of PC/ASA/PMMA

\begin{tabular}{|c|c|c|c|c|}
\hline $\begin{array}{c}\text { Amount of } \\
\text { ABS-g-MAH (g) }\end{array}$ & $\begin{array}{c}\text { Flexural } \\
\text { strength/MPa }\end{array}$ & $\begin{array}{c}\text { Tensile } \\
\text { strength/MPa }\end{array}$ & Shore D hardness & $\begin{array}{c}\text { Notched impact } \\
\text { strength/kJ*m }{ }^{-2}\end{array}$ \\
\hline 0 & 41.7 & 50.8 & 71 & 40.5 \\
\hline 3 & 40.6 & 48.7 & 71 & 38 \\
\hline 6 & 39.3 & 46.7 & 70 & 41.25 \\
\hline 9 & 40.9 & 50 & 70 & 47.4 \\
\hline 12 & 42.3 & 52 & 69 & 43.3 \\
\hline
\end{tabular}

The ABS-g-MAH is less rigid than PC, and it penetrated into each regions of different components in the blends and lowered its intermolecular forces, therefore the addition of ABS-g-MAH lead the blends lose some tensile strength ${ }^{[13]}$. However, the addition of ABS-g-MAH significantly improved both flexural and impact strength of PC/ASA/PMMA blends, this improving activities could be attributed to the structure and characteristics of ABS-g-MAH ${ }^{[14]}$.

The ABS-g-MAH has flexible anhydride groups ( $\mathrm{C}=\mathrm{O}$ double bonds) that could contribute to improve the blend toughness ${ }^{[15,16]}$. In addition, as the compatibilizer, ABS-g-MAH could reduce and component separation and connect each phases and improve the blend homogeneity. When 
the external stress was performed on blends, the stress could transfer to each component and the stress concentration was reduced.

The hardness of polymer blends were not largely modified by ABS-g-MAH, because the hardness of a polymer is related to the itsr relaxation behaviors, the ABS-g-MAH generally affected on the interface of two polymer phases rather than each polymer chains so that it didn't change the relaxation times of each polymer segments ${ }^{[17]}$, the hardness were not significantly affected.

In this study, it was found that the over addition of ABS-g-MAH could worsen the mechanical properties of the blends. This effects might be caused by two possibilities: 1. Too much ABS-g-MAH could form a new separated phase (micelle) and the regularity and homogeneity of polymer blend decreased; 2. Extra ABS-g-MAH could reduce the blend crystallinity and orientation extent ${ }^{[18]} ; 3$. The anhydride groups on ABS-g-MAH might react with ester groups of polycarbonate, under high temperature ans shearing (during injection molding) the anhyride groups might decompose thermally and affect on mechanical performances ${ }^{[19]}$.

The Figure 3 are storage modulus and loss modulus of PC/ASA/PMMA blends. The storage modulus of each blend has a critical point, which was in the range of 50 to $90^{\circ} \mathrm{C}$ and this point was the glass transition temperature of ABS or polycarbonate resin. The addition of ABS-g-MAH could improve the storage modulus, as the ABS-g-MAH had a smaller size and larger specific surface area, it could increase the viscosity between the interfaces, which makes segment movement more difficult. In addition, the loss modulus started changing at $50^{\circ} \mathrm{C}$ and it changed significantly in the range of $50-70^{\circ} \mathrm{C}$ and $90-100^{\circ} \mathrm{C}$, this was caused by the relaxations of different segments belong to different polymer components.

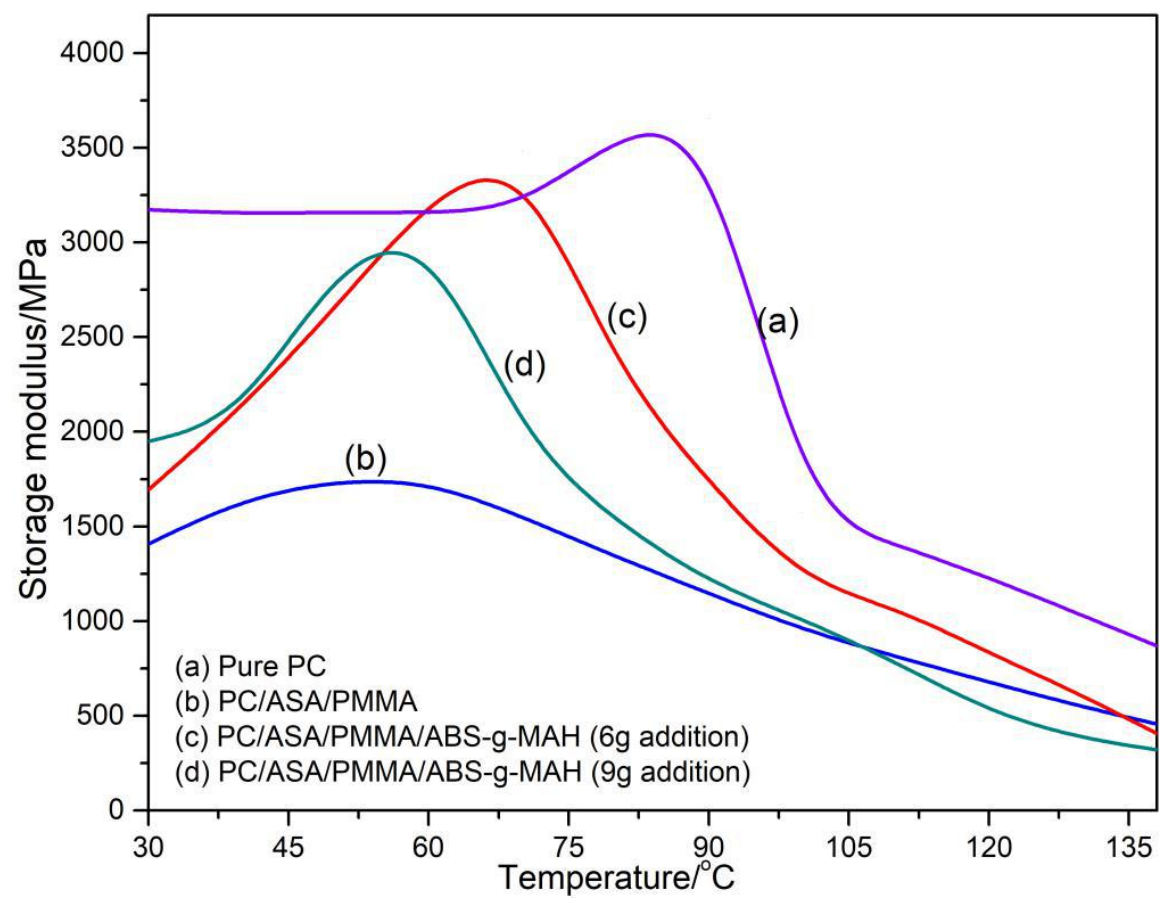

Fig.3(a). Storage modulus of PC/ASA/PMMA 


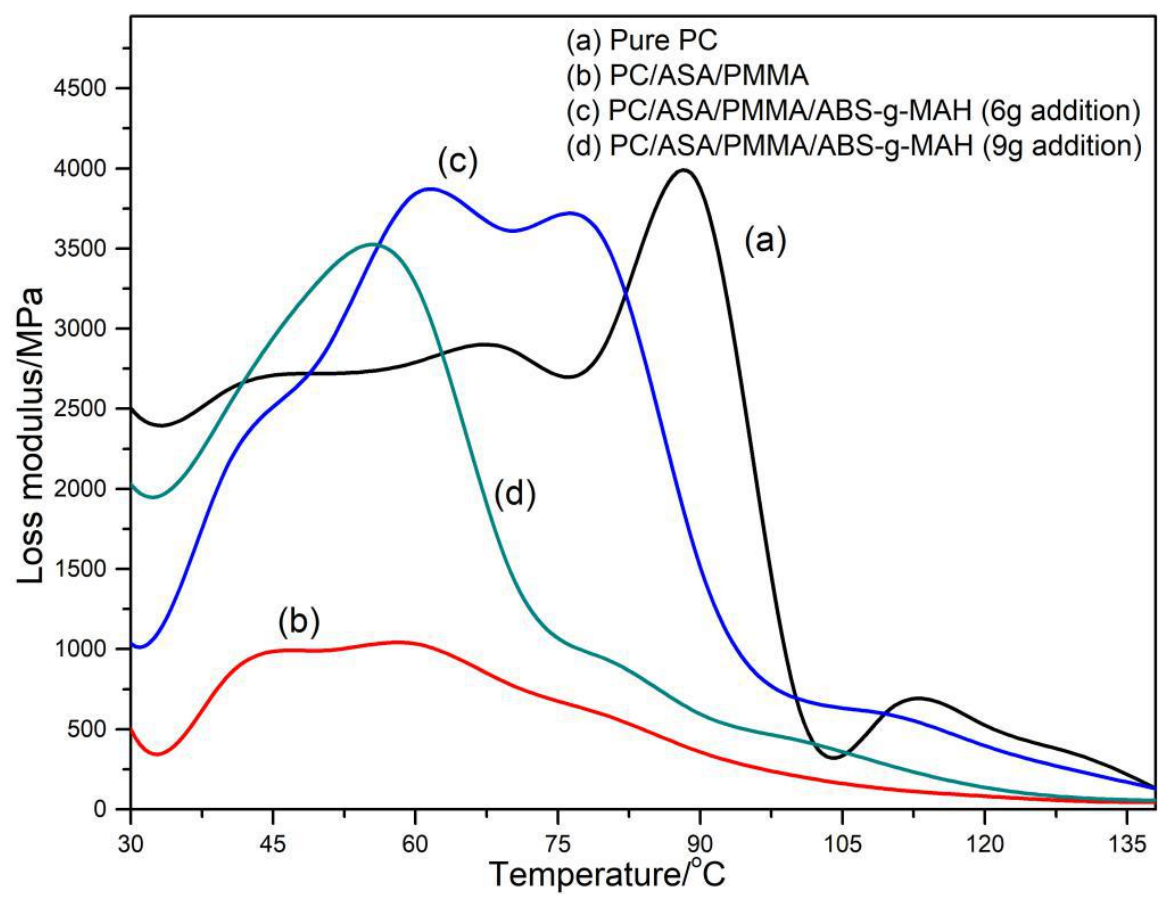

Fig.3(b). Loss modulus of PC/ASA/PMMA

\subsection{Thermal properties}

Figure 4 showed the DSC curves of PC/ASA/PMMA. Without ABS-g-MAH, the PC/ASA/PMMA showed two endothermic peaks compared to pure $P C$, which means the blend had two glass transition point and the higher point belonged to the PC phase. With ABS-g-MAH, only one glass transition point appeared, this indicated that the ABS-g-MAH promoted the binding between each phases ${ }^{[20]}$. Besides, the addition of $A B S-g-M A H$ lead the glass transition point of PC/ASA/PMMA a little bit higher than the unmodified one, this is because with ABS-g-MAH, the PC phase restricted the segment movement so more energies and free volume had to be consumed to initiate the glass transition ${ }^{[21]}$.

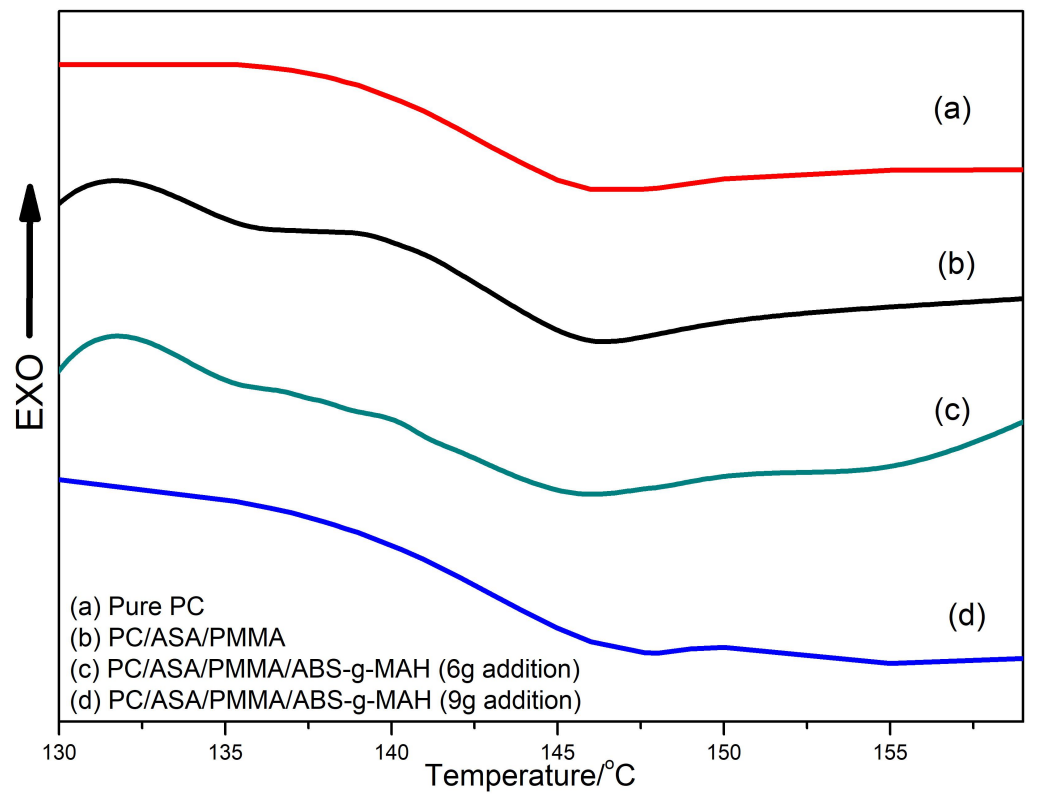

Fig.4. DSC curves of PC/ASA/PMMA 
Table 3 indicated that the blends modified by ABS-g-MAH had a lower Vicat softening point, this might be explain by the structure of ABS-g-MAH, the flexible 1,3-butadiene components softened the polymer blends

Table 3. Vicat softening temperatures of PC/ASA/PMMA blends

\begin{tabular}{|l|l|}
\hline Sample & Vicat softening point $/{ }^{\circ} \mathrm{C}$ \\
\hline $\mathrm{PC}$ & 151 \\
\hline PC/ASA/PMMA & 145 \\
\hline PC/ASA/PMMA/ABS-g-MAH (3g addition) & 143 \\
\hline PC/ASA/PMMA/ABS-g-MAH (6g addition) & 142 \\
\hline PC/ASA/PMMA/ABS-g-MAH (9g addition) & 141 \\
\hline PC/ASA/PMMA/ABS-g-MAH (12g addition) & 139 \\
\hline
\end{tabular}

Figure 4 and table 4 are the thermal stabilities of polymer blends tested by TGA. Compared to pure PC, the blend had a lower thermal stability, its initial decomposition temperature and $50 \%$ decomposition temperature decreased largely. The ABS-g-MAH lead the situation worse, because its butadiene segments/chains are extremely sensitive to thermal oxidation ${ }^{[22]}$, and under higher temperature, the anhydride groups could react with ester groups on PC and generate bubbles and small molecule products, these also accelerated the thermal decomposition.

Table 4. Thermal decomposition temperatures of blends

\begin{tabular}{|c|c|c|c|}
\hline Samples & $\begin{array}{c}\text { Residue } \\
\text { content/\% }\end{array}$ & $\begin{array}{c}\text { Initial decomposition } \\
\text { temperature } /{ }^{\circ} \mathrm{C}\end{array}$ & $\begin{array}{c}50 \% \text { decomposition } \\
\text { temperature/ }{ }^{\circ} \mathrm{C}\end{array}$ \\
\hline $\mathrm{PC}$ & 18.32 & 490.66 & 532 \\
\hline $\mathrm{PC} / \mathrm{ASA} / \mathrm{PMMA}$ & 9.68 & 407.48 & 423.67 \\
\hline $\mathrm{PC} / \mathrm{ASA} / \mathrm{PMMA} / \mathrm{ABS}-\mathrm{g}-\mathrm{MAH}$ (6g addition) & 9.13 & 407.47 & 419.2 \\
\hline $\mathrm{PC} / \mathrm{ASA} / \mathrm{PMMA} / \mathrm{ABS}-\mathrm{g}-\mathrm{MAH}$ (9g addition) & 7.87 & 407.2 & 416.34 \\
\hline
\end{tabular}

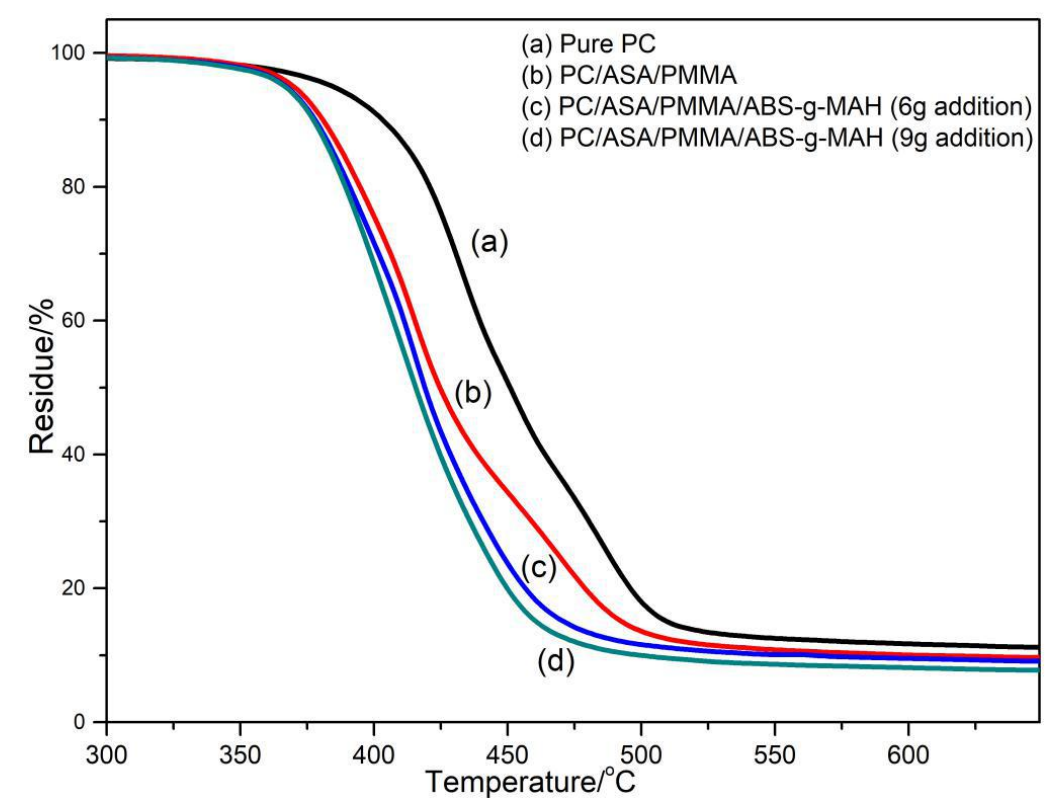

Fig.4. TGA curves of PC/ASA/PMMA 


\section{Conclusion}

In this study, different PC/ASA/PMMA blends were prepared and effect ABS-g-MAH on morphologies and properties of PC/ASA/PMMA blends were examined. The results showed that ABS-g-MAH could affect on the blends by different approaches, especially it could promote compatbilities and impact strengths but also reduce the tensile strength and thermal stabilities. In general, its structure flexibility, functional groups, chemical characteristics and usage amounts were innegligible. In order to obtain expected products, the usage of ABS-g-MAH is supposed to be carefully controlled.

\section{Acknowledgment}

This study was financially supported by Natural Science Foundation of China (50873049).

\section{Reference}

1. Ye Han, Zhi-Xin Tai, Chao Zhou, Ming-Yao Zhang, Hui-Xuan Zhang, Feng-Qi Liu. Influence of blend composition on the mechanical properties and morphology of PC/ASA/SAN ternary blends. Polymer Bulletin. 2009.62: 855-866.

2. Wei Zhang, Shuangjun Chen, Jun Zhang. Influence of blend composition on mechanical properties of ASA/SAN binary blends. Journal of Thermoplastic Composite Materials. 2011.26(3): 322-335.

3. Pulak Datta, Chandan Guha, Gautam Sarkhel. Study of mechanical, thermal, morphological, and process rheology of acrylonitrile styrene acrylate (ASA) $/ \mathrm{Na}^{+1}$ poly(ethylene-co-methacrylic acid) ionomer blend. Polymer Science and Enigeering. 2015.1571-1579.

4. R. Navarro, J. Lo'pez, F. Parres, S. Ferra'ndiz. Process behavior of compatible polymer blends. Journal of Applied Polymer Science. 2012.124: 2485-2493.

5. Ruogu Tang. Effects of sulfur curing systems on mechanical properties of NR/SBR rubber blends. 2019. ChemRxiv. Preprint.

6. Xiaoxia Cai, Bingpeng Li, Yi Pan, Guozhang Wu. Morphology evolution of immiscible polymer blends as directed by nanoparticle self-agglomeration. Polymer. 2012.53(1): 259-266.

7. Roberta K. Sadi, Rafael S. Kurusu, Guilhermino J. M. Fechine, Nicole R. Demarquette. Compatibilization of polypropylene/ poly(3-hydroxybutyrate) blends. Journal of Applied Polymer Science. 2012.123: 3511-3519.

8. Sung Kyu Kim, Robin Jang, Woo Nyon Kim. Effects of compatibilizer on the mechanical, rheological, and shape memory behaviors of poly(lactic acid) and poly(MnBM) blends. 2019.136: 48591.

9. Harekrishna Panigrahi, P.R. Sreenath, Anil K. Bhowmick, K.Dinesh Kumar. Unique compatibilized thermoplastic elastomer from polypropylene and epichlorohydrin rubber. Polymer. 2019.183(21): 121866.

10. Hisham A. Essawy, Salwa H. El-Sabbagh, Magda E. Tawfik, Guy Van Assche, Ahmed Barhoum. Assessment of provoked compatibility of NBR/SBR polymer blend with montmorillonite amphiphiles from the thermal degradation kinetics. Polymer Bulletin. 2018.75(4): 1417-1430.

11. Bo Wu, Xian Zheng, Jinhua Leng, Bin Yang, Xian Chen, Bobing He. Compatibilization of ethylene/maleic anhydride/glycidyl methacrylate terpolymer for poly(phenylene sulfide)/poly(amide-66) blends. Journal of Applied Polymer Science. 2012.124:, 325-332.

12. Xiangfu Zhang, Yusheng Chen, Yong Zhang, Zonglin Peng, Yinxi Zhang, Wen Zhou. Effects of 
ABS-g-MAH on mechanical properties and compatibility of ABS/PC alloy. 2001.81: 831-836.

13. A. Farzadfar, S. Nouri Khorasani. Effect of EVA-g-MAH on Mechanical Properties of PC/ABS Blends. Polymer-Plastics Technology and Engineering. 2012.51(14): 1424-1426.

14. M.I. Triantou, P.A. Tarantili. Studies on morphology and thermomechanical performance of ABS/PC/Organoclay hybrids. Polymer Composite. 2014.35: 1395-1407.

15. J.H. Kim, C.K. Kim. Changes in the Interfacial Properties of PC/SAN Blends with Compatibilizer. Journal of Applied Polymer Science. 2003.89: 649-2656.

16. Haiyun $\mathrm{Ma}$, Lifang Tong, Zhongbin Xu, Zhengping Fang. Clay network in ABS-graft-MAH nanocomposites: Rheology and flammability. Polymer Degradation and Stability. 2007.92(8): 1439-1445.

17. Adam Danch, Wieslaw W. Sułkowski, Marek Moczyński, Agnieszka Radon, Franz Stelzer, Stefan Jurga. Structural relaxation and morphology of the rubber-urethane composites. Journal of Applied Polymer Science. 2004.94: 1186-1193.

18. Weibing $\mathrm{Xu}$, Guodong Liang, Hongbo Zhai, Shupei Tang, Guopei Hang, Wei-Ping Pan. Preparation and crystallization behaviour of PP/PP-g-MAH/Org-MMT nanocomposite. European Polymer Journal. 2003. 39(7): 1467-1474.

19. Weibing Xu, Guodong Liang, Wei Wang, Shupei Tang, Pingsheng He, Wei-Ping Pan. Poly(propylene)-poly(propylene)-grafted maleic anhydride-organic montmorillonite (PP-PP-g-MAH-Org-MMT) nanocomposites. II. Nonisothermal crystallization kinetics. Journal of Applied Polymer Science. 2003.88: 3093-3099.

20. FP Tseng, JJ Lin, CR Tseng, FC Chang. Poly(oxypropylene)-amide polypropylene as novel compatibilizers for PP and PA6 blends. Polymer. 2001. 42(2): 713-716.

21. Biju John, K.T. Varughese, Zachariah Oommen, Sabu Thomas. Melt rheology of HDPE/EVA blends: The effects of blend ratio, compatibilization, and dynamic vulcanization. Polymer Engineering and Science. 2010. 50(4): 665-676.

22. Chenxu Wu, Jian Zhou, Xuefei Li, Lei Li. Properties and microstructure of polybutylene terephthalate/polycarbonate alloy plastics. CISEC Journal. 2010.61(6): 1571-1576. 